\title{
Transfer of diffuse astronomical light and airglow in scattering Earth atmosphere
}

\author{
S. S. Hong ${ }^{1}$, S. M. Kwon², Y.-S. Park ${ }^{3}$, and C. Park ${ }^{1}$ \\ ${ }^{1}$ Department of Astronomy, Seoul National University, Seoul, Korea \\ ${ }^{2}$ Department of Science Education, Kangwon National University, Korea \\ ${ }^{3}$ Korea Astronomy Observatory, Yusong-Ku, Daejon, Korea
}

(Received October 7, 1997; Revised February 24, 1998; Accepted February 27, 1998)

\begin{abstract}
To understand an observed distribution of atmospheric diffuse light (ADL) over an entire meridian, we have solved rigorously, with the quasi-diffusion method, the problem of radiative transfer in an anisotropically scattering spherical atmosphere of the earth. In addition to the integrated starlight and the zodiacal light we placed a narrow layer of airglow emission on top of the scattering earth atmosphere. The calculated distribution of the ADL brightness over zenith distance shows good agreement with the observed one. The agreement can be utilized in deriving the zodiacal light brightness at small solar elongations from the night sky brightness observed at large zenith distances.
\end{abstract}

\section{Introduction}

One of the most difficult tasks in reducing the night sky observations to the zodiacal light is to remove the atmosphererelated diffuse light. Since air molecules and aerosols divert light from off the telescope axis into the telescope beam via multiple scattering, the brightness observed at a sky point has contributions from not only the point itself but also the off-axis region. Although many attempts have been made to bypass the difficulties, they are only partially successful. What makes problem worse is that brightnesses of the zodiacal light (ZL), the integrated starlight (IS), and the airglow (AG) are all comparable to each other.

It is important to know how much of the off-axis light gets into the beam, particularly at large zenith distances. Staude (1975) investigated how the light from Milky Way and the zodiacal cone are transported through the earth atmosphere, with the AG being left out. Limiting his calculations to single scattering component, he could properly take into account for the atmospheric sphericity and the localized brightness enhancements of the Milky Way and ZL. To estimate the contribution from the multiple scattering he relaxed the sphericity condition and made use of the calculations done for a planeparallel atmosphere of Rayleigh scattering. In this study we will limit ourselves to those parts of the night sky where the localized nature of the Milky Way and ZL would not matter much, but fully solve the problem of radiative transfer in an anisotropically scattering, spherical atmosphere.

\section{Questions to Answer}

If we denote by $\operatorname{BS}(\alpha, \delta ; \zeta)$ the observed brightness of sky point $(\alpha, \delta)$ through the earth atmosphere at zenith distance $\zeta$, BS is given by the sum $\mathrm{BS}=\mathrm{ST}+\mathrm{IS}+\mathrm{ZL}+\mathrm{AG}$, where $\mathrm{ST}$ is the observed brightness of resolved starlight. From star catalogs one may obtain the brightness $\mathrm{ST}^{\circ}(\alpha, \delta)$ of the resolved stars outside the atmosphere, and ST is given by

Copy right (C) The Society of Geomagnetism and Earth, Planetary and Space Sciences (SGEPSS); The Seismological Society of Japan; The Volcanological Society of Japan; The Geodetic Society of Japan; The Japanese Society for Planetary Sciences.
$\mathrm{ST}^{\circ}(\alpha, \delta) \exp [-\tau(\zeta)]$. Here, $\tau(\zeta)$ is the extinction optical depth over the atmospheric path at $\zeta$.

One may not use the same exponential factor to remove IS $(\alpha, \delta ; \zeta)$, because it is an extended source. Formally the IS is resulted from a "convolution" of the brightness $\operatorname{IS}^{\circ}(\alpha, \delta)$ before entering the atmosphere with a "transfer" function $\mathrm{T}_{\mathrm{atm}}(\Delta \alpha, \Delta \delta ; \zeta)$ of the earth atmosphere:

$$
\operatorname{IS}(\alpha, \delta ; \zeta)=\operatorname{IS}^{\circ}\left(\alpha^{\prime}, \delta^{\prime}\right) \otimes \mathrm{T}_{\mathrm{atm}}\left(\alpha^{\prime}-\alpha, \delta^{\prime}-\delta ; \zeta\right) .
$$

A similar description

$$
\mathrm{AG}(\zeta)=\mathrm{AG}^{\circ}\left(\zeta^{\prime}\right) \otimes \mathrm{T}_{\mathrm{atm}}\left(\zeta^{\prime} ; \zeta\right)
$$

can be made for the AG. Using the Pioneers, Weinberg actually observed $\mathrm{IS}^{\circ}$ (Toller, 1981). On the contrary, the airglow brightness $\mathrm{AG}^{\circ}$ on top of the earth scattering atmosphere is not known observationally. One usually models it by a van Rhijn type function $\mathrm{AG}_{\mathrm{Rhijn}}^{\circ}(\zeta)$ (Roach and Gordon, 1972).

Details of the transfer function and nature of the convolution depend, in a complicated way, on the atmospheric properties and also the outside source itself. Thus the description done in Eqs. (1) and (2) is of no practical use. There have been many attempts to devise a workable means for effecting the convolution (Elsässer and Haug, 1960; Weinberg, 1963; Dumont, 1965; Hong et al., 1985; Kwon, 1990; Kwon et al., 1991). (Some of these were critically compared to each other by Staude (1975).) Dumont proposed to use different extinction optical depths for point and extended sources. He argued that the optical depth for extended source should be less than the one for stars. By taking an effective extinction optical depth $\tau_{\text {eff }}(\zeta) \equiv f \tau(\zeta)$, with $f$ being less than 1 , one may compensate the off-axis light contribution. Kwon (1990) took this approach, and approximated the result of convolution as

$$
\mathrm{IS}(\alpha, \delta ; \zeta) \simeq \operatorname{IS}^{\circ}(\alpha, \delta) \exp \left[-\tau_{\mathrm{eff}}(\zeta)\right]
$$

for the integrated starlight and

$$
\mathrm{AG}(\zeta) \simeq \mathrm{AG}^{\circ}(\zeta) \exp \left[-\tau_{\mathrm{eff}}(\zeta)\right]
$$




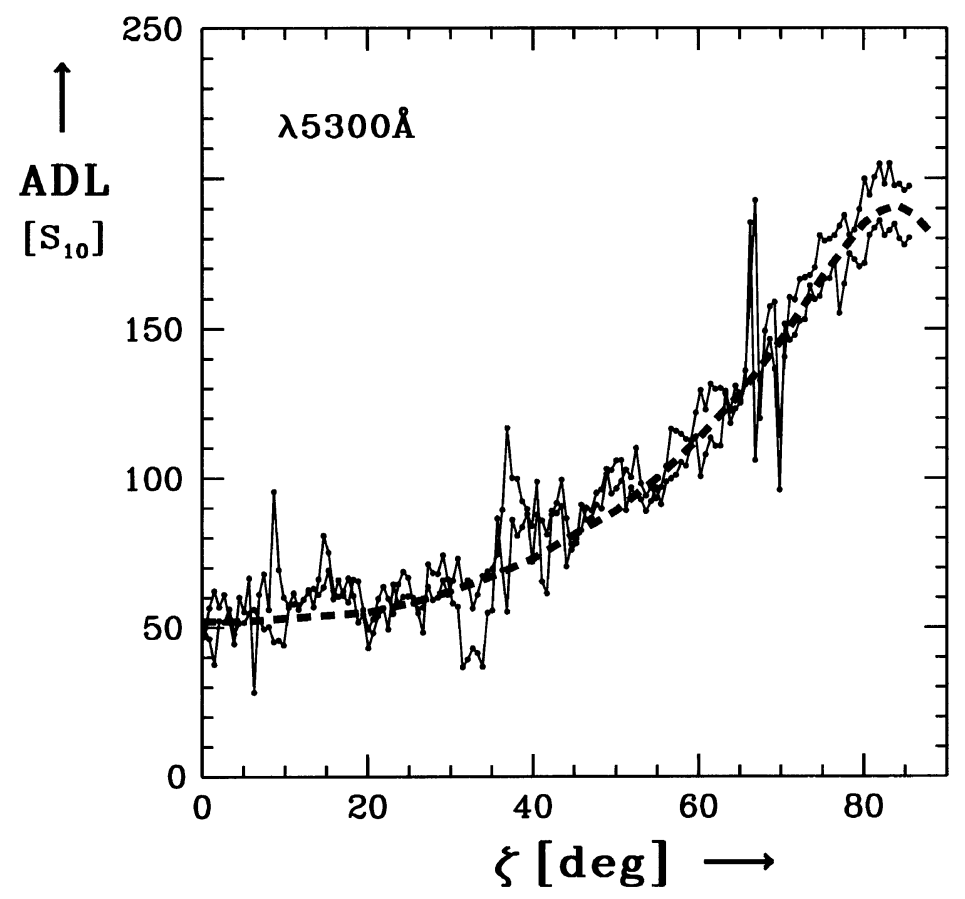

Fig. 1. Observed brightness of the ADL is plotted against zenith distance.

for the airglow. He then removed ST, IS and AG from BS.

We now ask ourselves the following two questions: (1) How accurately does the effective $\tau$ factor account for the off-axis contribution? (2) Is the van Rhijn function a good model for the airglow emission?

\section{Atmospheric Diffuse Light over the Meridian}

On the night of August 21/22, 1968 Weinberg and Mann scanned the meridian at Mt. Haleakala, Hawaii from the zenith to horizon 11 times at each of the wavelengths $5080 \AA$ and $5300 \AA$. The meridian scan is an excellent data base for answering the questions. Kwon (1990) took the Pioneer data for IS ${ }^{\circ}$, and the zodiacal light of Levasseur-Regourd and Dumont (1980) for $\mathrm{ZL}^{\circ}$. He carefully measured the zenith extinction optical depth $\tau(\zeta=0) \simeq 0.16$, and empirically determined $f$ to be about 0.75 . By using the $\tau_{\text {eff }}$ in the exponential factor, he subtracted, from the observed meridian brightness $\operatorname{BS}(\zeta)$, the sum of ST, IS and ZL:

$$
\begin{aligned}
\operatorname{ADL}(\zeta) \equiv & \operatorname{BS}(\zeta)-\mathrm{ST}^{\circ} \exp [-\tau(\zeta)] \\
& -\left[\mathrm{IS}^{\circ}+\mathrm{ZL}^{\circ}\right] \exp \left[-\tau_{\mathrm{eff}}(\zeta)\right] .
\end{aligned}
$$

We call this residual as the atmospheric diffuse light ADL.

The thin solid line in Fig. 1 presents the ADL brightness at $5300 \AA$ as a function of zenith distance. To avoid crowdedness only two scans are shown here. The spiky features are due to an inaccurate subtraction of bright stars. The thick dashed line shows an average of all the 11 scans. If the questions we raised have affirmative answers, the resulting ADL would have only the airglow component. It is then interesting to see how closely the $\operatorname{ADL}(\zeta)$ resembles its theoretical counterpart $\mathrm{AG}_{\mathrm{Rhijn}}^{\circ}\left(\zeta^{\prime}\right) \otimes \mathrm{T}_{\mathrm{atm}}\left(\zeta^{\prime} ; \zeta\right)$.

Since there isn't any easy way to perform the operation symbolized by $\otimes$, we turn to a numerical means. As far as the radiation field impinging upon the earth atmosphere has an azimuthal symmetry with respect to outward normal, the quasi-diffusion method based on ray-tracing technique (Cassinelli and Hummer, 1971; Hummer and Rybicki, 1971; Leung, 1975, 1976) is an efficient tool for getting numerical solutions to the problem. We have developed a quasidiffusion radiative code, which uses the Henyey-Greenstein function as the scattering phase function. (Details of the code are beyond the scope of this paper, and will be reported elsewhere.) With the code we calculated the ADL brightness as a function of $\zeta$.

A combination of the Rayleigh scattering and two HenyeyGreenstein functions with asymmetry factor 0.82 and -0.55 was used for the mean scattering phase function (McCartney, 1976). We took 0.25 for surface reflection coefficient, and 0.76 for particle albedo. For the density distribution we used an exponentially decreasing function with scale height $H=$ $8 \mathrm{~km}$. For the airglow we placed at height $h=80 \mathrm{~km}$ an airglow emitting layer, within which the density varies as $\exp \left[-(r-h)^{2} / W^{2}\right]$ with $W=40 \mathrm{~km}$.

We placed the earth within an isotropic radiation field of unit brightness, and calculated the brightness $I\left(\zeta ; R_{E}\right)$ that would be observed by an observer on the earth surface. The solid line in Fig. 2 shows the $\zeta$-dependence of the brightness and the dashed one does the same of $\exp [-\tau(\zeta)]$. If there were no scattered photons or the atmosphere were of purely absorbing nature, the solid and dashed lines would overlap each other. The difference, $\mathrm{I}\left(\zeta ; \mathrm{R}_{\mathrm{E}}\right)-\exp [-\tau(\zeta)]$, shown by short dash-dotted line represents the scattered component, which dominates the brightness near the horizon.

To have an idea about the $f$ value and its dependence on aerosol properties, we have calculated $\mathrm{I}\left(\zeta ; \mathrm{R}_{\mathrm{E}}\right)$ with varying particle albedo $\varpi$ and asymmetry factor $g$. In Fig. 3 we then plotted $2.5 \log \mathrm{I}\left(\zeta ; \mathrm{R}_{\mathrm{E}}\right)$ against $\tau(\zeta) / \tau(0)$, which is an 


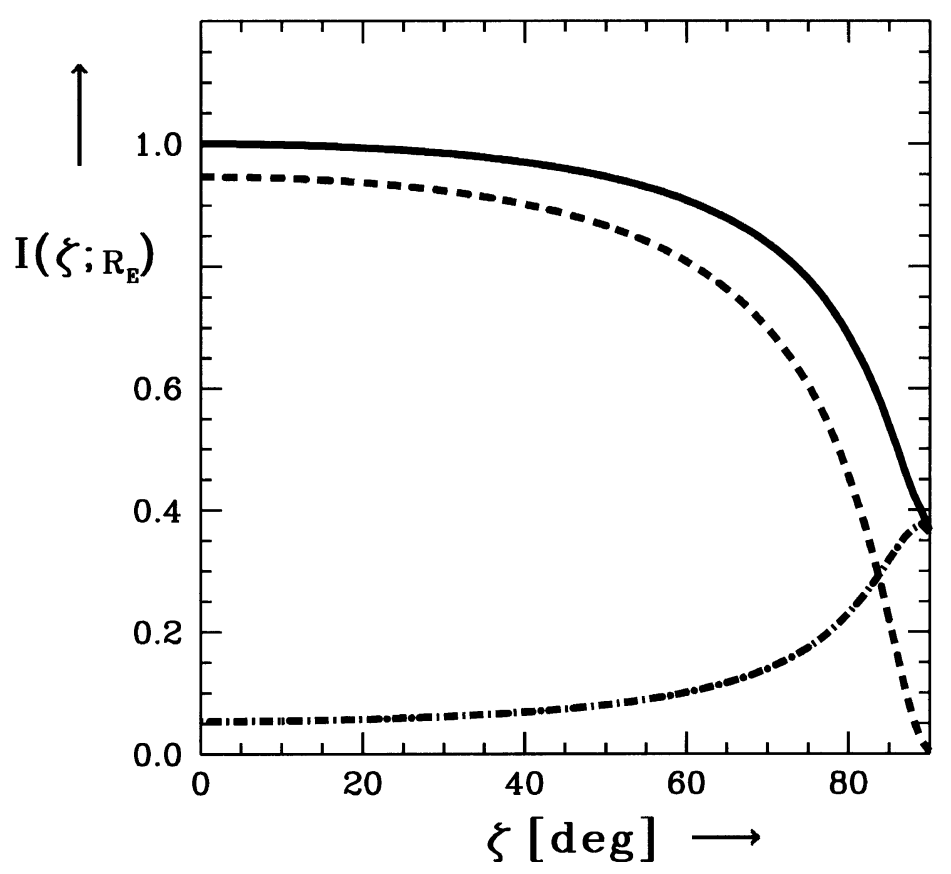

Fig. 2. When an isotropic incident radiation reaches the ground, its brightness decreases towards the horizon. The short dash-dotted line represents the multiply scattered component.

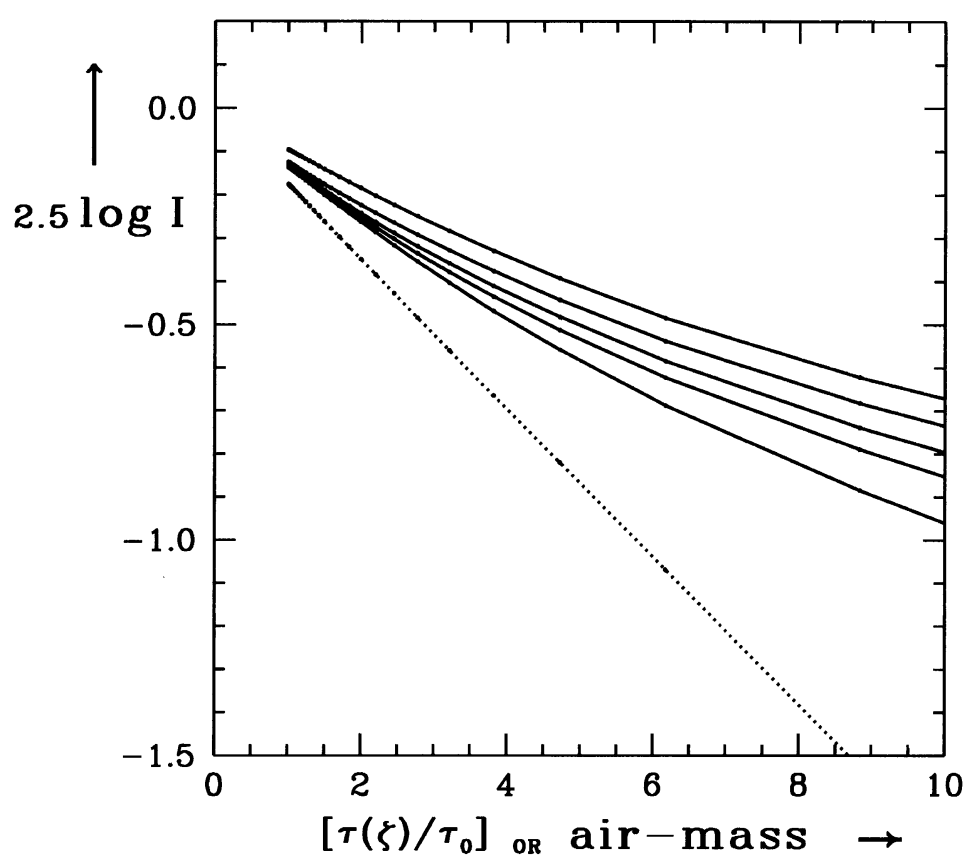

Fig. 3. Observed magnitude is plotted against air-mass. The dotted line is for the case of pure absorption. The five solid lines from bottom to top are for $g=0.9,0.8,0.7,0.5$, and 0.0 , and albedo is fixed at 0.76 .

equivalent of the usual air-mass $x$. The curves can be fitted to straight lines in the range $x \leqq 2$. From their slopes we determined the effective optical depth $\tau_{\text {eff }}(0)$ at the zenith and hence $f$. Figure 4 summarizes the dependence of $f$ on $\varpi$ and $g$. As $\varpi$ decreases and $g$ increases, $f$ increases towards 1 . And $f$ is almost independent of $\tau(\zeta=0)$. It is interesting to note that the empirically determined 0.75 is not an unacceptable value for $f$. However, it is clear from this figure that the effective $\tau$ approach breaks down at zenith distances larger than about $60^{\circ}$.

Placing an emitting layer in the atmosphere, we have calculated the airglow brightness. The long dash-dotted line in Fig. 5 represents the brightness distribution of the airglow $\mathrm{AG}(\zeta)$ that would be observed on the earth surface. By ad- 


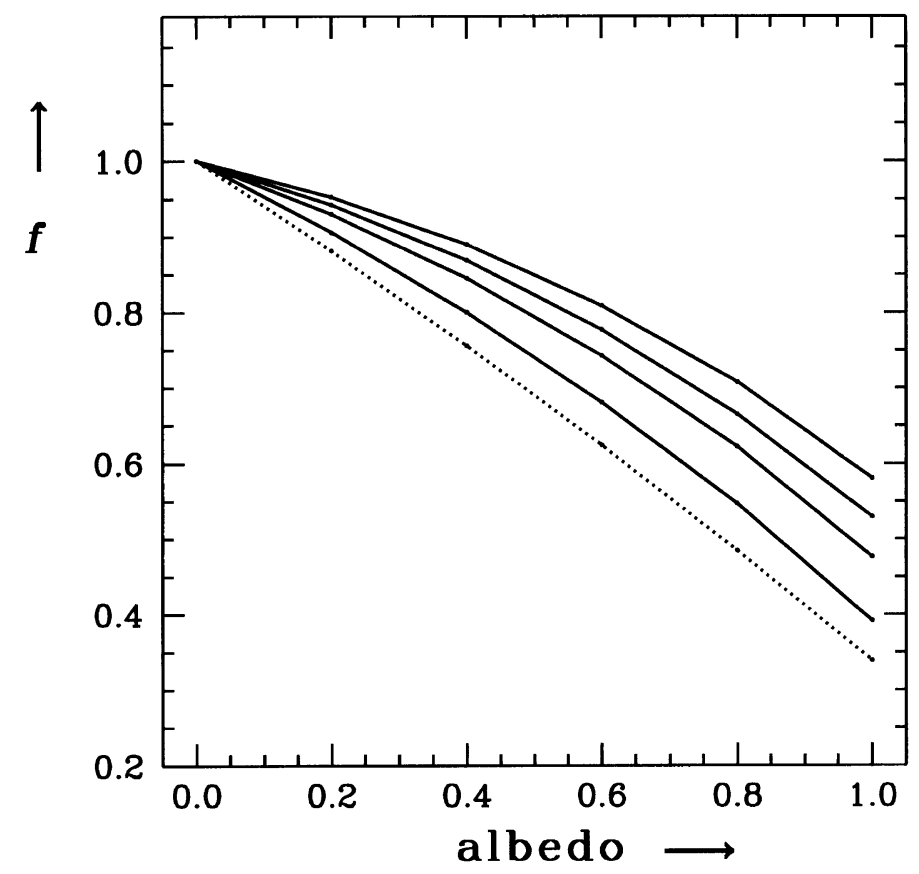

Fig. 4. Ratio $\tau_{\text {eff }}(0) / \tau(0)$ is plotted against albedo for 5 values of $g$. From lower to upper curves $g$ takes $0.0,0.5,0.7,0.8$, and 0.9 . The zenith optical depth is kept at 0.16 .

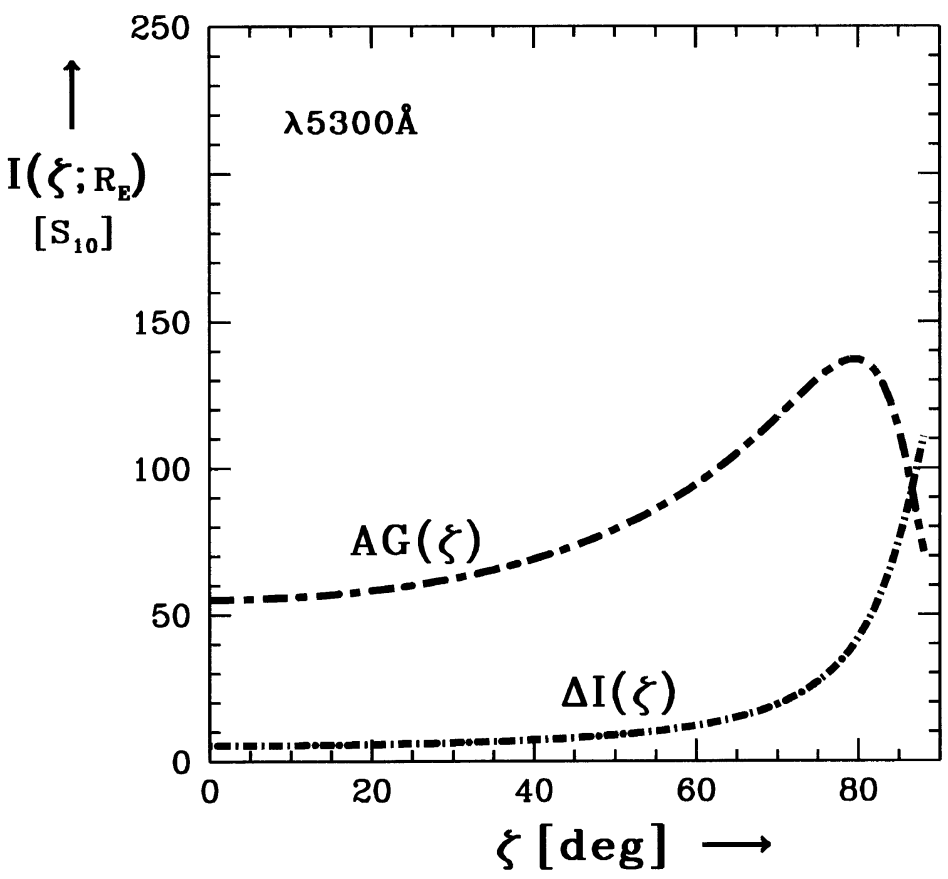

Fig. 5. Distribution of the airglow intensity as observed on the earth surface is shown by long dash-dotted line. Short dash-dotted line is for the residual intensity of IS and ZL.

justing the zenith brightness, we tried to match the observed $\operatorname{ADL}(\zeta)$ distribution with the calculated $\operatorname{AG}(\zeta)$. As can be seen from the two dash-dotted lines in Fig. 6, the $\operatorname{AG}(\zeta)$ alone doesn't seem to reproduce the observed ADL over the entire meridian. This is understandable, because the effective $\tau$ approach doesn't fully compensate the multiply scattered components of the IS and ZL. The short dash-dotted line in
Fig. 5 represents the difference

$$
\Delta \mathrm{I}(\zeta) \equiv \mathrm{I}\left(\zeta ; R_{\mathrm{E}}\right)-\exp \left[-\tau_{\mathrm{eff}}(\zeta)\right]
$$

where we have used the solid line in Fig. 2 for $\mathrm{I}\left(\zeta\right.$; $\left.\mathrm{R}_{\mathrm{E}}\right)$. The solid line in Fig. 6 is a weighted sum of $\mathrm{AG}(\zeta)$ and $\Delta \mathrm{I}(\zeta)$. The weighting is done in such a way that out of the total zenith brightness $415 \mathrm{~S}_{10}$, the airglow comprises $55 \mathrm{~S}_{10}$ with 


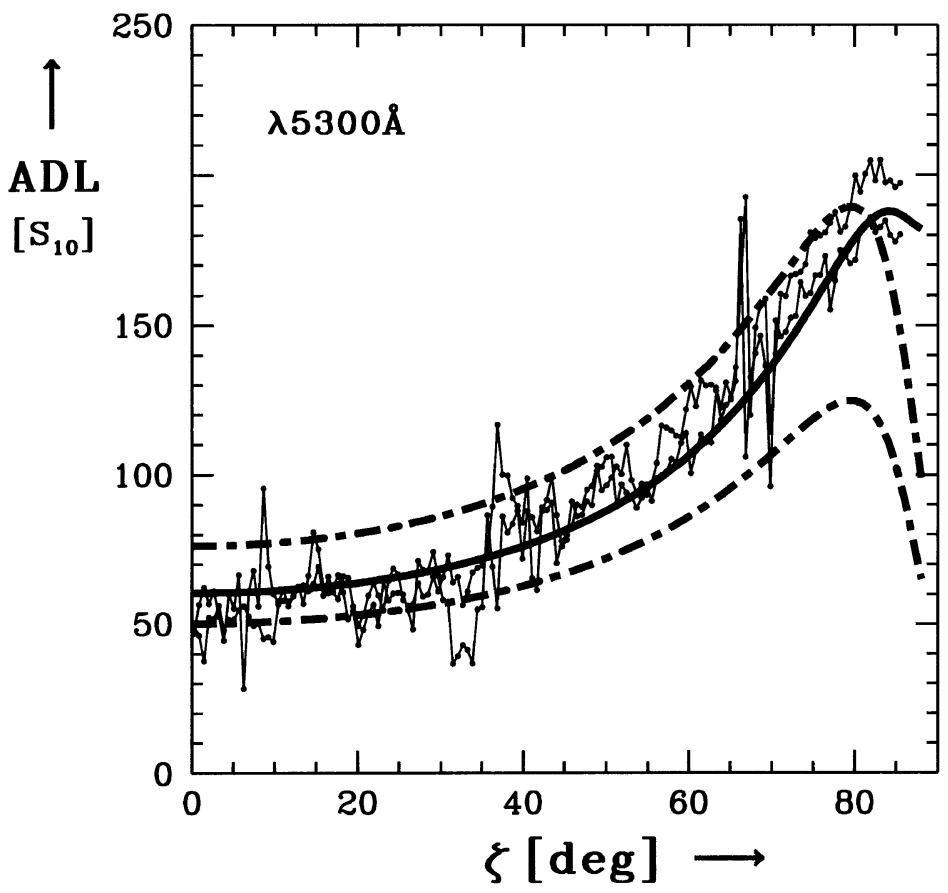

Fig. 6. Two dashed lines are trials to fit the ADL by airglow alone, and solid line is sum of the AG and the residual of IS + ZL.

the remaining $360 \mathrm{~S}_{10}$ coming from the IS and ZL. Over the whole night the zenith brightness of the $\mathrm{ZL}^{\circ}$ varied from 60 to $100 \mathrm{~S}_{10}$.

\section{Conclusion}

From this study we may draw following conclusions: The effective $\tau$ approach is useful in the reduction of night sky observations only at small zenith distances. The van Rhijn type function is a reasonable model for estimating the airglow brightness over a large range of zenith distance. We have theoretically reproduced the observed distribution of the atmospheric diffuse light over the entire meridian by fully solving the problem of radiative transfer in an anisotropically scattering atmosphere of the earth. This type of theoretical understandings will provide us with a means for reducing the night sky brightness observed near horizon to the zodiacal light brightness at small elongations.

Acknowledgments. This study was supported in part by the Korean Ministry of Education, Basic Science Research Institute grant No. BSRI-97-5411, and SMK by the travel fund of Kangwon National University, Republic of Korea.

\section{References}

Cassinelli, J. P. and D. G. Hummer, Radiative Transfer in Spherically Symmertric Systems - II, The Non-Conservative Case and Linearly Polarized Radiation, MNRAS, 154, 9-21, 1971.

Dumont, R., Separation des composantes atmosphérique interplanetaire et stellaire du ciel nocture a $5000 \AA$ A: Application à la photometrie de la lumiere zodiacale et du Gegenschein, Ann. d'Astrophys., 28, 265-320, 1965.

Elsässer, H. and U. Haug, Über eine lichtelektrische Flächenphotometrie der stüdlichen und nördlichen Milchstrasse in zwei Farben und die Struktur des galaktischen Systems, Z. Astrophys., 50, 121, 1960

Hong, S. S., N. Y. Misconi, M. H. H. van Dijk, J. L. Weinberg, and G. N. Toller, A Search for Small Scale Structures in the Zodiacal Light, in Properties and Interactions of Interplanetary Dust, Proc. of IAU Colloq. \#85, edited by R. H. Giese and P. L. Lamy, 33pp., Dordrecht, Reidel, 1985 .

Hummer, D. G. and G. B. Rybicki, Radiative Transfer in Spherically Symmertric Systems. The Conservative Grey Case, MNRAS, 152, 1-19, 1971

Kwon, S. M., A Fine Resolution Study of the Zodiacal Light, Ph.D. Thesis, Seoul National University, 1990.

Kwon, S. M., S. S. Hong, and J. L. Weinberg, Temporal and Spatial Variations of the Atmospheric Diffuse Light, in Origin and Evolution of Interplanetary Dust, Proc. of IAU Colloq. \#126, edited by A. C. LevasseurRegourd and H. Hasegawa, 179pp., Dordrecht, Reidel, 1991.

Leung, C. M., Radiation Transport in Dense Interstellar Dust Clouds. I. Grain Temperature, Astrophys. J., 199, 340-360, 1975.

Leung, C. M., Radiation Transport in Dense Interstellar Dust Clouds. II. Infrared Emission from Molecular Clouds Associated with H II Regions, Astrophys. J., 209, 75-93, 1976.

Levasseur-Regourd, A. C. and R. Dumont, Absolute Photometry of Zodiacal Light, Astron. Astrophys., 84, 277-279, 1980.

McCartney, E. J., Optics of the Atmosphere, 408pp., John Wiley, New York, 1976.

Roach, F. E. and J. L. Gordon, The Light of the Night Sky, 54pp., Dordrecht, Reidel, 1972

Staude, H. J., Scattering in the Earth's Atmosphere: Calculations for Milky Way and Zodiacal Light as Extended Sources, Astron. Astrophys., 39, 325-333, 1975.

Toller, G. N., A Study of Galactic Light, Extragalactic Light, and Galactic Structure Using Pioneer 10 Observations of Background Starlight, Ph.D. Thesis, State University of New York at Stony Brook, 1981.

Weinberg, J. L., Photoelectric Polarimetry of the Zodiacal Light at $\lambda 5300$, Ph.D. Thesis, University of Colorado, 1963.

S. S. Hong (e-mail: sshong@astroism.snu.ac.kr), S. M. Kwon (e-mail smkwon@ceres.kangwon.ac.kr), Y.-S. Park (e-mail: yspark@nanul.issa. re.kr), and C. Park (e-mail: chanpk@astro.snu.ac.kr) 